\title{
Recomendaciones sobre diagnóstico, manejo y estudio de la infección del tracto urinario en pediatría. Rama de Nefrología de la Sociedad Chilena de Pediatría. Parte 1
}

\section{Recommendations on diagnosis, management and study of the urinary tract infection in pediatrics. Nephrology Branch of the Chilean Society of Pediatrics. Part 1}

\author{
Hevia J. Pilar ${ }^{a}$, Alarcón O. Claudia ${ }^{b}$, González C. Claudia ${ }^{c}$, Nazal Ch. Vilma $^{\text {, }}$ Rosati M. María Pía ${ }^{\mathrm{a}}$
}

\author{
${ }^{a}$ Hospital San Juan de Dios, Santiago, Chile

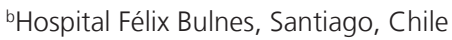 \\ cHospital Sótero del Río, Santiago, Chile \\ ¿Clínica Quilín Hospital Clínico Universidad de Chile, Santiago, Chile
}

Recibido el 28 de mayo del 2019; aceptado el 25 de septiembre del 2019

¿Qué se sabe del tema que trata este estudio?

La infección urinaria (ITU) es una patología prevalente en pediatría y su manejo ha cambiado considerablemente en los últimos años.

\section{¿Qué aporta este estudio a lo ya conocido?}

Actualiza las recomendaciones para el diagnóstico clínico y de laboratorio, incluyendo los puntos de corte para el diagnóstico bacteriológico en muestras tomadas por cateterismo vesical. Se dan pautas sobre tratamiento antibiótico y su duración en las distintas formas de presentación de la ITU.

\begin{abstract}
Resumen
La infección del tracto urinario (ITU) es una de las infecciones bacterianas mas frecuentes en la edad pediátrica, pero su diagnóstico y manejo se pueden ver complicados por lo inespecífico de sus síntomas y signos de presentación, la dificultad en la interpretación de los exámenes, especialmente en niños mas pequeños, y por un pronóstico respecto a daño renal muchas veces incierto. En los últimos años, se ha evidenciado una modificación significativa en el enfoque diagnostico y terapéutico de esta patología, surgiendo la necesidad de actualizar las recomendaciones previas. El propósito de esta revisión es contribuir a reducir la variabilidad de la práctica clínica en el manejo de ITU en la población pediátrica, mejorando la detección y manejo de la patología estructural y otros factores de riesgo de daño renal, evitando acciones innecesarias en aquellos niños con bajo riesgo. En esta primera parte, se presentan las recomendaciones en cuanto a diagnóstico y manejo de la ITU en pediatría. En la segunda parte se detalla su estudio, prevención y seguimiento.
\end{abstract}

Palabras clave: infección del tracto urinario; pielonefritis aguda; cicatriz renal; reflujo vésico-ureteral; niños

Correspondencia:

Hevia J. Pilar

pilarheviaj@gmail.com 


\begin{abstract}
Urinary tract infection (UTI) is one of the most frequent bacterial infection in pediatrics. However, its diagnosis and management can be complicated due to the nonspecific clinical presentation, the difficulty of exams interpretation, especially in younger children, and an uncertain prognosis regarding renal damage. In recent years, significant worldwide change has come in treatment, diagnosis, and images studies, we have decided to update the current recommendations on UTI management published by the Pediatric Nephrology branch of Chilean Pediatrics Society in previous years. The purpose of these recommendations is to reduce the variability of clinical practice in management of UTI in our pediatric population, favoring diagnostic and therapeutic interventions in the most appropriate way, improving detection and management of structural pathology and other risk factors of renal damage, avoiding unnecessary actions in children with low risk. This first part includes diagnosis and treatment recommendations of urinary tract infection in pediatric age. In the second part the study, prevention and monitoring of urinary tract in pediatric age is detailed.
\end{abstract}

\section{Keywords:}

urinary tract infection; acute pyelonephritis; renal scars; vesicoureteral reflux; children

\section{Introducción}

En las últimas décadas se ha producido un cambio en el curso clínico de la infección del tracto urinario (ITU) como resultado de la introducción del tratamiento antibiótico, mejoría de los métodos diagnósticos y accesibilidad a la atención de salud. Además, existe mayor conocimiento de los mecanismos de daño renal y las evidencias actuales han demostrado que la mayoría de los niños que han tenido ITU no tienen complicaciones a largo plazo. Todo esto ha contribuido a la falta de certeza sobre la manera más apropiada de diagnosticar la ITU y si los estudios y seguimiento están justificados o no.

La ITU es una de las infecciones bacterianas más frecuentes en la edad pediátrica y su manejo se ve influido por lo inespecífico de sus síntomas y signos de presentación, la dificultad en la interpretación de los exámenes, especialmente en niños más pequeños, y por la incertidumbre que su pronóstico conlleva.

En los últimos años, y como contribución de la Medicina basada en evidencia, se ha producido a nivel mundial una modificación significativa en la conducta e intervenciones que hasta la fecha se venían realizando $^{1-3}$, surgiendo la necesidad de actualizar las recomendaciones sobre manejo de la ITU que la Rama de Nefrología de la Sociedad Chilena de Pediatría había publicado en años anteriores ${ }^{4}$.

El propósito de estas recomendaciones es contribuir a reducir la variabilidad de la práctica clínica en el manejo de ITU en la población pediátrica, favoreciendo que las intervenciones diagnósticas y terapéuticas se realicen de la forma más adecuada posible, mejorando la detección y manejo de la patología estructural y otros factores de riesgo de daño renal, pero evitando acciones innecesarias en aquellos niños de bajo riesgo.

La metodología empleada se basó en una búsqueda actualizada de la literatura utilizando los buscadores
Pubmed y LILACS, con especial énfasis en revisiones sistemáticas, y de las principales guías clínicas internacionales publicadas. El grupo de trabajo, constituido para este fin, analizó la evidencia, y las recomendaciones entregadas fueron consensuadas por todos sus integrantes. Posteriormente se envió a los miembros de la rama de nefrología infantil para su conocimiento y aprobación antes de la publicación.

Estas recomendaciones están dirigidas a todos los profesionales que participan en el diagnóstico, tratamiento y estudio de los pacientes pediátricos con ITU desde 1 mes de edad hasta los 18 años. Se excluyen a los recién nacidos, a pacientes con catéteres urinarios, vejiga neurogénica, uropatías significativas preexistentes, enfermedad de base como síndrome nefrótico, inmunosuprimidos, pacientes en unidades de cuidados intensivos y mujeres adolescentes sexualmente activas que presentan ITU recurrente.

En esta primera parte, se presentan las recomendaciones en cuanto a diagnóstico y manejo de la ITU en pediatría.

\section{Epidemiología}

La infección del tracto urinario constituye una de las infecciones bacterianas que se observan con mayor frecuencia en el lactante y escolar. Su incidencia acumulativa es difícil de estimar dado la heterogeneidad de los estudios epidemiológicos. Alrededor de 1/10 niñas y $1 / 30$ niños han tenido ITU a la edad de 16 años. Antes de los 2 años de vida, el 2,1\% de las niñas y 2,2\% de los niños han presentado al menos un episodio de ITU $^{2}$.

La prevalencia de ITU varía con la edad, sexo y presencia o no de circuncisión. En lactantes menores de 2 años con fiebre, ésta es de 7\%, y en niños de 2 a 19 años con síntomas urinarios y/o fiebre, es de 7,8\%. La pre- 
valencia es más alta en los lactantes menores febriles no circuncidados y en niñas menores de $1 \mathrm{año}{ }^{5}$.

Los pacientes que han presentado una primera ITU tienen alto riesgo de recurrencia. La incidencia de ITU recurrente (ITUR) en niños y niñas con tracto urinario normal varía entre 19 y $41 \%$ en los distintos estudios. En los menores de 1 año es de cerca del 30\%, y más del 90\% recurre dentro del primer año de evolución, especialmente, los primeros meses ${ }^{1}$. Los factores de riesgo de recurrencia de ITU son: primera ITU antes de los 6 meses de edad, historia familiar de ITU, reflujo vésicoureteral (RVU) dilatado y disfunción vejiga-intestino ${ }^{2}$.

\section{Definición y clasificación de la infección del tracto urinario}

La ITU se define como la colonización bacteriana del tracto urinario, que puede comprometer desde la vejiga hasta el parénquima renal, asociado a leucocituria y sintomatología clínica variable.

La presentación clínica puede $\operatorname{ser}^{1,4,6}$ :

- Cistitis o ITU baja: infección limitada a la vejiga y a la uretra. Los pacientes refieren síntomas secundarios a inflamación local como disuria, polaquiuria, urgencia, hematuria y dolor suprapúbico en niños que controlan esfínter.

- ITU alta o pielonefritis aguda (PNA): infección bacteriana del tracto urinario superior que compromete el parénquima renal. El síntoma principal es la fiebre, otros síntomas comunes son la irritabilidad, dolor abdominal y vómitos. Los niños mayores pueden presentar fiebre y dolor lumbar, aunque a veces es solo fiebre sin foco.

- Bacteriuria asintomática (BA): presencia de bacterias con recuentos significativos en muestras repetidas de orina durante seguimiento después de una ITU o en controles de salud, en ausencia de síntomas sistémicos o urinarios. Puede haber o no alteraciones en el sedimento de orina (leucocituria $)^{7}$. Es mas frecuente en niñas en edad escolar y carece de transcendencia clínica.

- ITU recurrente: definida como 3 o más infecciones urinarias bajas, 2 o más PNA o 1 pielonefritis más 1 infección urinaria baja en 1 año.

- ITU atípica o complicada: ITU alta que evoluciona en forma tórpida. Su identificación es importante pues requiere un manejo y estudio individualizado. En este cuadro clínico, además de los síntomas sistémicos, se asocian elementos que sugieren alteraciones anatómicas o funcionales de la vía urinaria tales como:

- Chorro urinario débil.

- Masa abdominal o vesical.

- $\quad$ Aumento de creatinina.
- Septicemia.

- No respuesta al tratamiento antibiótico apropiado en las primeras 48 horas.

- Infección por agente no E. coli.

\section{Diagnóstico}

El diagnóstico de ITU debe plantearse frente a una historia y examen físico sugerente, asociado a un examen de orina compatible y se confirma con un urocultivo positivo (Figura 1).

\section{Clínica}

La sospecha clínica de ITU en la población pediátrica se fundamenta en una serie de signos y síntomas más o menos específicos (tabla 1). Requiere confirmación con exámenes de laboratorio ya que la clínica posee baja capacidad discriminatoria.

En niños menores de 24 meses el síntoma principal es la presencia de fiebre sin foco.

Con el fin de evitar realizar exámenes innecesarios, que en este grupo etario significa realización de exámenes por sondeo o punción vesical, y a la vez reducir el riesgo de retraso de inicio de terapia antibiótica en los pacientes que lo requieran, se han estudiado diferentes variables clínicas que permiten diferenciar aquellos lactantes con fiebre que tienen bajo o alto riesgo de ITU. Esa así como, en lactantes febriles de 2 a 24 meses, se recomienda realizar examen de orina frente a las siguientes situaciones clínicas ${ }^{3}$ :

a) Niñas con 2 o más de los siguientes factores: edad $<12$ meses, fiebre por más de 48 horas, fiebre sin causa aparente y fiebre $>$ o igual a $39^{\circ} \mathrm{C}$.

b) En niños circuncidados con fiebre $>0$ igual a $39^{\circ}$ por más de 24 h y sin causa aparente.

c) En niños no circuncidados basta presencia de fiebre $>$ o igual de $39^{\circ} \mathrm{C}$ por 24 horas, sin otros factores de riesgos adicionales.

En niños mayores y adolescentes, se deben realizar exámenes de orina si presentan síntomas de disuria, urgencia, polaquiuria, orina turbia, hematuria o dolor abdominal o lumbar, con o sin fiebre. También en niños de cualquier edad con fiebre sin causa aparente, y que en la anamnesis destaque: antecedentes de ITU previa, anormalidad de la vía urinaria como hidronefrosis, RVU, displasia renal, vejiga neurogénica, disfunción vesical, o en niños no verbales con retraso cognitivo.

En todo niño con ITU es importante descartar mediante la anamnesis y el examen físico los factores de riesgo de ITU y patología subyacente de importancia (tabla 2). 


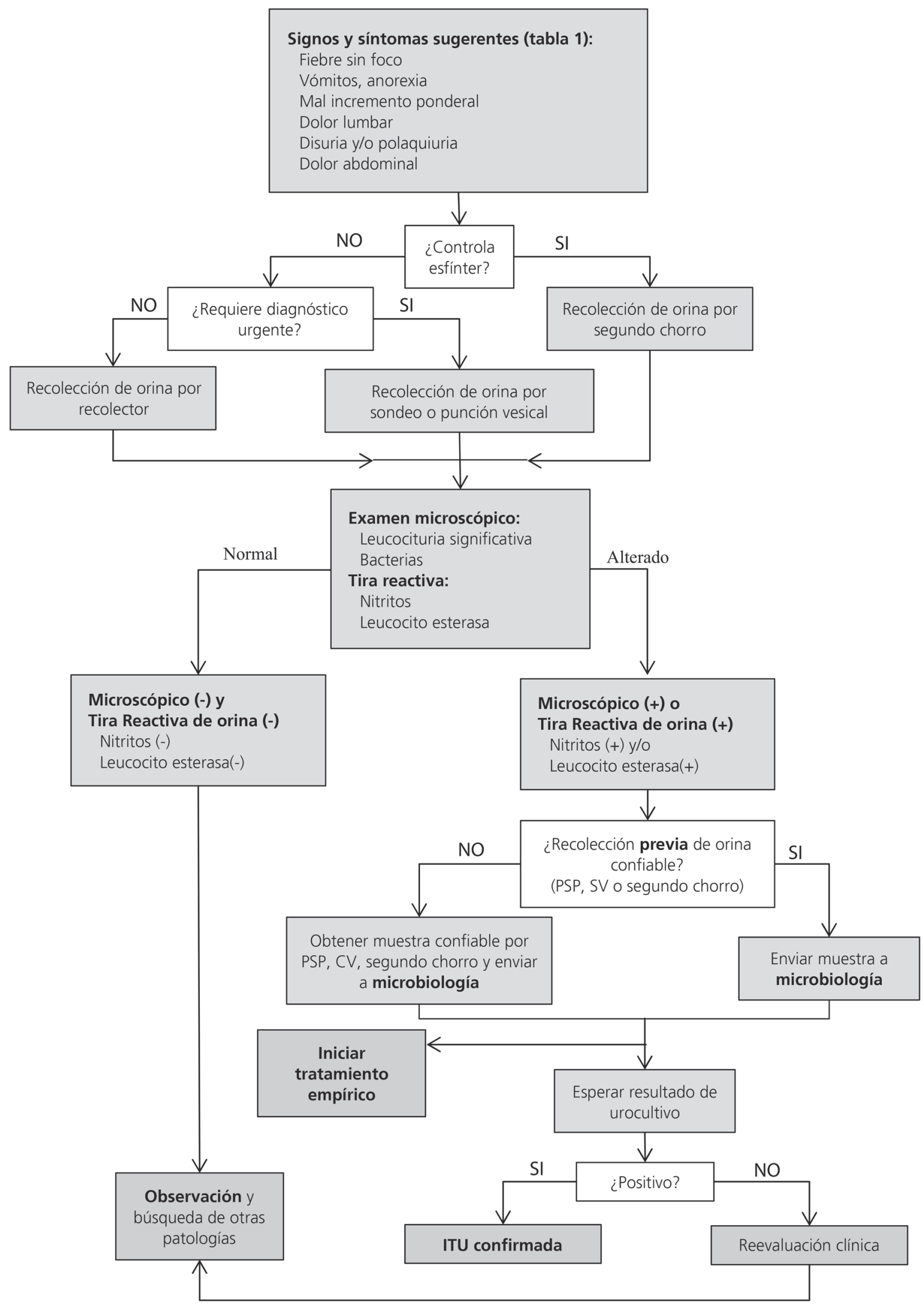

Figura 1. Algoritmo diagnóstico de ITU1'. 
Tabla 1. Síntomas y signos en lactantes y niños con ITU²

\begin{tabular}{|c|c|c|c|c|}
\hline \multirow{2}{*}{\multicolumn{2}{|c|}{ Grupo de edad }} & \multicolumn{3}{|c|}{ Síntomas y signos } \\
\hline & & Mas comunes & & Menos comunes \\
\hline Lactante $<3$ meses & & $\begin{array}{l}\text { Fiebre } \\
\text { Vómitos } \\
\text { Letargia } \\
\text { Irritabilidad }\end{array}$ & $\begin{array}{l}\text { Rechazo alimentación } \\
\text { Retraso crecimiento }\end{array}$ & $\begin{array}{l}\text { Dolor abdominal } \\
\text { Hematuria } \\
\text { Ictericia } \\
\text { Orina mal olor }\end{array}$ \\
\hline \multirow[t]{2}{*}{$\begin{array}{l}\text { Lactantes y niños } \\
>3 \text { meses }\end{array}$} & Preverbal & Fiebre & $\begin{array}{l}\text { Dolor abdominal } \\
\text { Vómitos } \\
\text { Rechazo alimentación } \\
\text { Dolor lumbar }\end{array}$ & $\begin{array}{l}\text { Letargia } \\
\text { Irritabilidad } \\
\text { Hematuria } \\
\text { Orina mal olor } \\
\text { Retraso crecimiento }\end{array}$ \\
\hline & Verbal & $\begin{array}{l}\text { Polaquiuria } \\
\text { Disuria }\end{array}$ & $\begin{array}{l}\text { Vaciamiento disfuncional } \\
\text { Incontinencia } \\
\text { Dolor abdominal } \\
\text { Dolor lumbar }\end{array}$ & $\begin{array}{l}\text { Fiebre } \\
\text { Malestar } \\
\text { Vómitos } \\
\text { Hematuria } \\
\text { Orina mal olor } \\
\text { Orina turbia }\end{array}$ \\
\hline
\end{tabular}

\section{Examen de orina}

El diagnóstico de ITU es bacteriológico, por lo que es fundamental tener una muestra de orina fiable para evitar errores diagnósticos y procedimientos innecesarios.

\section{Toma de muestra}

La elección del método de recolección de orina va a depender de la capacidad de control de la micción y de la situación clínica del paciente (en cuanto a la urgencia diagnóstica y de tratamiento) (figura 1).

Para los niños con control de micción se recomienda recoger la muestra de orina de la mitad del chorro miccional (orina de $2^{\circ}$ chorro). La muestra de orina debe ser tomada por personal entrenado, previo lavado de genitales con jabón suave y considerar una buena hidratación del paciente previo a la recolección ${ }^{1}$.

En el caso de niños sin continencia urinaria y que requieren un diagnóstico y/o tratamiento inmediato, se sugiere que la muestra de orina se realice por punción suprapúbica o cateterismo vesical. La elección del método dependerá del nivel de entrenamiento y del entorno asistencial ${ }^{1-4,8}$. Habitualmente se prefiere el cateterismo vesical, que puede realizarse tanto en servicios de urgencia como en pacientes hospitalizados. Es una técnica molesta para el niño, pero con bajo riesgo de complicaciones ${ }^{9}$, aunque también es susceptible de contaminación, con tasas reportadas entre un 9 y $12 \%$. Para disminuir el riesgo de contaminación, se deben eliminar los primeros mililitros de orina obtenida por la sonda y utilizar una sonda nueva si el primer intento no fue exitoso ${ }^{1,3,10}$. La punción suprapúbica, técnica de referencia para el cultivo de orina, es invasiva, molesta
Tabla 2. Factores de riesgo de patología nefrourológica subyacente ${ }^{1,2}$

Anamnesis y examen físico dirigido a evaluar:

- Chorro urinario débil

- Antecedentes de ITU previa

- Antecedentes de fiebre recurrente sin foco

- Diagnóstico prenatal de anomalías del tracto urinario

- Antecedentes familiares de reflujo vésico-ureteral u otra patología renal

- Constipación

- Disfunción miccional

- Globo vesical

- Masa abdominal

- Lesiones de médula espinal

- Mal desarrollo pondoestatural

- Hipertensión arterial

y requiere personal entrenado, por lo que está generalmente restringida a pacientes con fimosis severa, sinequia vulvar o infecciones o malformaciones de genital externos. En caso de optar por ésta, se recomienda que sea bajo visión ecográfica.

Las bolsas recolectoras tienen un alto riesgo de contaminación, con tasas de falsos positivos inaceptablemente elevadas (mayor al 70\%), por lo que no se recomiendan para hacer el diagnóstico de ITU. Su mayor utilidad está en el caso que el examen de orina sea normal, permitiendo descartar ITU por su alto valor predictivo negativo. Se pueden utilizar en pacientes no continentes que no requieren un diagnóstico y terapia inmediatos, pero si la muestra está alterada, se debe 
confirmar con una nueva muestra de orina obtenida por sondeo o punción vesical antes de iniciar el tratamiento antibiótico. La muestra de orina debe procesarse antes de 4 horas; de no ser posible, se debe refrigerar entre $2-8^{\circ} \mathrm{C}$ inmediatamente tras su recogida por no más de 24 horas $^{1-4,8}$.

\section{Uroanálisis}

Es muy rara la presencia de ITU sin leucocituria (piuria). La leucocituria sin bacteriuria es inespecífica y puede estar presente en múltiples condiciones clínicas como fiebre, ejercicio intenso, vulvovaginitis, balanitis, glomerulonefritis, apendicitis, enfermedad de Kawasaki, etc. Por otro lado, la bacteriuria sin piuria orienta a contaminación externa, bacteriuria asintomática, o rara vez, infección muy reciente (antes del inicio de la inflamación $)^{3,10}$. Un uroanálisis alterado orienta al diagnóstico de ITU y permite iniciar tratamiento antibiótico precoz, antes del resultado del urocultivo, en pacientes con alta sospecha clínica. Debe realizarse en orina fresca recién emitida ${ }^{3}$. Comprende la tira reactiva y el examen microscópico de orina.

La tira reactiva analiza la presencia de nitritos y leucocito esterasa. Los nitritos positivos tienen alta especificidad pero baja sensibilidad (53\%), ya que se requieren a lo menos 4 horas de permanencia de la orina en vejiga para que el uropatógeno convierta los nitratos de la dieta a nitritos. Se observan falsos negativos en lactantes y niños pequeños que tienen vaciamiento vesical rápido, en ITU por uropatógenos que no reducen nitratos, como Pseudomonas aeruginosa, Enterococo spp, Staphylococcus saprophiticus y Candida spp, y ante la presencia de ácido ascórbico en la orina ${ }^{10,11}$.

La prueba de leucocito esterasa (LE) detecta la presencia de piuria o leucocitos en orina, reflejo de inflamación en la vía urinaria, lo que es esencial para establecer el diagnóstico diferencial de ITU vs contaminación o bacteriuria asintomática ${ }^{3,10,11}$.

El examen microscópico de orina (sedimento urinario) analiza la presencia de leucocitos y bacterias y se puede realizar en muestra de orina centrifugada o no centrifugada. Se considera leucocituria (piuria) significativa la presencia de mas de 10 leucocitos $/ \mathrm{mm}^{3}$ en orina no centrifugada (método hemocitométrico), y más de 5 leucocitos/campo o más de 25 leucocitos/uL en orina centrifugada (método estándar). Su realización consume más tiempo y requiere de personal adiestrado e instrumental adecuado.

La presencia combinada de LE y nitritos en la tira reactiva tiene una sensibilidad de $93 \%$ y especificidad de $72 \%$, por lo que se puede utilizar con seguridad para el diagnóstico biológico de la ITU, sobre todo en niños mayores de 2 años. En lactantes se sugiere realizar el examen microscópico además de la tira reactiva, ya que algunos estudios, con bajo número de pacientes, mos- trarían una menor validez de ésta, aunque probablemente tengan más relación con la calidad de la muestra que con el método ${ }^{1}$.

\section{Urocultivo}

El urocultivo se considera positivo o negativo en base al numero de unidades formadoras de colonias (UFC) que crecen en un medio de cultivo. El recuento de colonias considerado como significativo dependerá del método de recolección de la muestra: $\geq 50.000$ $\mathrm{UFC} / \mathrm{ml}$ en muestras por cateterismo vesical $\mathrm{y} \geq \mathrm{a}$ $100.000 \mathrm{UFC} / \mathrm{ml}$ si es por orina de $2^{\circ}$ chorro o bolsa recolectora (esta última, con alto riesgo de contaminación). En caso de punción vesical, basta el desarrollo de $1 \mathrm{UFC} / \mathrm{ml}$.

En pacientes con clínica sugerente (fiebre mas leucocituria) se puede considerar ITU con recuentos menores, entre 10.000 y $50.000 \mathrm{UFC} / \mathrm{ml}$, sobre todo cuando el uropatógeno es no E. coli, lo que está asociado a una baja respuesta inflamatoria ${ }^{3,10-12}$.

Sugieren contaminación en un urocultivo las siguientes situaciones:

- Muestra por bolsa recolectora.

- Crecimiento de más de un microorganismo.

- Crecimientos de comensales de la piel.

- Recuento de colonias menor a lo estandarizado.

- Urocultivo positivo sin leucocituria o con ex orina normal.

\section{Otros exámenes de laboratorio}

Si bien los exámenes de laboratorio como hemograma, VHS, PCR y procalcitonina orientan al diagnóstico de la localización de la ITU, no influyen en el manejo ni en la terapia antibiótica, por lo que no es imprescindible su realización de manera rutinaria ${ }^{1,2,8,13}$. Tampoco se requiere una creatinina plasmática en una ITU febril de evolución favorable.

Solo un 4-9\% de los lactantes con ITU febril cursan con bacteremia, por lo que se recomienda realizar hemocultivos solo en los grupos de riesgo, a saber: lactantes menores de 3 meses, niños con aspecto tóxico o séptico y niños con malformaciones del tracto urinario como obstrucción y $\mathrm{RVU}^{1,2,8,14}$.

\section{Tratamiento}

Los objetivos del tratamiento en la ITU son: obtener la mejoría clínica, erradicar la infección y minimizar el riesgo de secuelas a largo plazo ${ }^{1-4}$.

\section{Medidas generales}

Hidratación adecuada.

Paracetamol en caso de fiebre o dolor. No usar antiinflamatorios no esteroidales. 
Indicaciones de hospitalización: Restringida a niños con riesgo de bacteremia o que no puedan manejarse correctamente de manera ambulatoria ${ }^{1}$ (tabla 3 ).

\section{Tratamiento antibiótico empírico}

La elección del tratamiento dependerá de la resistencia antibiótica local ${ }^{15}$. Se recomienda no usar el mismo antibiótico que el paciente usaba para profilaxis ni el mismo que esté recibiendo por otra infección.

\section{ITU baja}

Alternativas: nitrofurantoína (NTF), cefalosporinas de $1^{\text {a }}$ generación, fosfomicina (en $>12$ años). El cotrimoxazol (CTX) es una buena alternativa, pero no puede utilizarse de manera empírica por el alto porcentaje de resistencia, considerar solo si el antibiograma lo permite. La duración del tratamiento es de 3-4 días (fosfomicina dosis única) ${ }^{2,16}$.

\section{ITU alta}

Puede tratarse por vía oral o parenteral, dependiendo de la edad y condiciones clínicas asociadas, ya que estudios han demostrado que no existen diferencias en cuanto a control clínico, recurrencias y cicatrices renales en DMSA 6 meses post infección ${ }^{1,17}$.

\section{Alternativas de tratamiento antibiótico empírico}

Paciente ambulatorio: cefalosporina de $2^{\mathrm{a}}$ o $3^{\mathrm{a}}$ generación vía oral, amikacina o ceftriaxona cada 24 hrs iv (en Servicio de Urgencia).

Paciente hospitalizado: Amikacina como $1^{\text {a }}$ elección, cefalosporinas de $2^{\mathrm{a}} \mathrm{o} 3^{\mathrm{o}}$ generación. El cambio a tratamiento oral podrá realizarse después de 24 horas
Tabla 3. Indicaciones de hospitalización ${ }^{1,2,4,6}$

Indicaciones de hospitalización

1. Edad menor de 3 meses

2. Aspecto tóxico o signos clínicos de sepsis

3. Mala tolerancia oral

4. Deshidratación

5. Mala respuesta clínica con tratamiento antibiótico adecuado

6. Dudas en el cumplimiento del tratamiento ambulatorio

7. Malformación del tracto urinario como displasia, uropatía obstructiva, RVU, riñón único

8. Inmunodeficiencia $1^{\mathrm{a}} \circ 2^{\mathrm{a}}$

9. Alteraciones electrolíticas o de la función renal

10. Indicaciones relativas: elevación importante de reactantes de fase aguda, ITU febril recurrente, lactante mayor de 3 meses con historia familiar de RVU o ecografía prenatal con hidronefrosis congénita

afebril y dependerá de la tolerancia oral y del informe del antibiograma ${ }^{3}$.

En el menor de 3 meses debe asociarse ampicilina al tratamiento antibiótico empírico por la posibilidad de infección por enterococo.

Evitar uso de aminoglicósidos en pacientes con compromiso de función renal.

La continuación del tratamiento dependerá del antibiograma. Preferir el antibiótico de espectro más reducido para disminuir riesgo de resistencia antibiótica y selección de cepas. Ej: cefalosporina de $1^{\text {a }}$ generación o CTX. La NTF no puede utilizarse en ITU alta ya que no se concentra en el parénquima renal.

Se debe reevaluar la respuesta clínica a las 48 horas.

Tabla 4. Manejo agudo y tratamiento ${ }^{1,2,21}$

Cuadro clínico Manejo agudo y tratamiento

Paciente $<3$ meses y/o pacientes con signos de infección grave, antecedentes de uropatía obstructiva, RVU alto grado

\section{Pielonefritis Aguda:}

Niños con bacteriuria mas fiebre $\geq 38^{\circ} \mathrm{C}$

Niños con bacteriuria, fiebre $<38^{\circ} \mathrm{C}$

y dolor lumbar o puño percusión +

Cistitis o ITU baja:

Niños con síntomas urinarios, bacteriuria y sin síntomas sistémicos

Nefronia o absceso renal
Referencia a servicio de urgencia

Examen de orina confiable y hemocultivos

Hospitalización

Tratamiento EV hasta 24 horas afebril y buena tolerancia oral (mínimo 5 días si bacteremia)

Tiempo de tratamiento 7 a 10 días (10 días si bacteremia)

Examen de orina confiable, urocultivo si no se ha realizado

Tratamiento antibiótico oral por 7-10 días

Si no es posible tratamiento oral, considerar tratamiento EV inicial por 1 a 3 días y luego continuar con vía oral

Control médico a las 24- 48 horas

Tratamiento oral por 3 a 4 días

Tratamiento 21 d, inicialmente biasociado (cefalosporina $3^{\text {a }}+$ aminoglicósido). Completar por vía oral 
Duración tratamiento: 7-10 días ${ }^{2,3}$ (tabla 4). No se recomienda realizar urocultivo intra o post tratamiento, a menos que exista sintomatología urinaria posterior a 48 horas de iniciado el tratamiento o exista dudas en cuanto la adherencia a éste.

En caso de ITU con bacteremia, se sugiere tratamiento antibiótico endovenoso por un mínimo de 5 días, hasta 24-48 h afebril, con mejoría clínica y adecuada tolerancia al antibiótico oral, y completar 10 días de tratamiento en total ${ }^{18-21}$.

En nefronia lobar o absceso, el tratamiento debe ser por 3 semanas $^{1,21}$, inicialmente tratamiento antibiótico endovenoso biasociado (cefalosporina $3^{\mathrm{a}}+$ aminoglicósido) y completar tratamiento por vía oral de acuerdo al urocultivo, una vez que se obtenga la mejoría clínica.

La bacteriuria asintomática en niños no debe tratarse con antibióticos, dado que se aumenta la resistencia antibiótica, no tiene riesgo de secuelas y tiene alta probabilidad de recaídas ${ }^{2,7}$.

\section{Conflicto de intereses}

Los autores declaran no tener conflicto de intereses.

\section{Referencias}

1. Grupo de trabajo de la Guía de Práctica Clínica sobre Infección del Tracto Urinario en la Población Pediátrica. Guía de Práctica Clínica sobre Infección del Tracto Urinario en la Población Pediátrica. Plan de Calidad para el Sistema Nacional de Salud del Ministerio de Sanidad, Política Social e Igualdad. Instituto Aragonés de Ciencias de la Salud. Guías Práctica Clínica en el SNS.2011;I+CN No2009/01.

2. National Collaborating Centre for Women's and Children's Health (UK). Urinary Tract Infection in Children: Diagnosis, Treatment and Long-term Management [Internet]. London: RCOG Press; 2007. Available from: https://www. ncbi.nlm.nih.gov/books/NBK50606.

3. Urinary Tract Infection: Clinical Practice Guideline for the Diagnosis and Management of the Initial UTI in Febrile Infants and Children 2 to 24 Months. Pediatrics [Internet]. 2011;128(3):595610. Available from: http://pediatrics. aappublications.org/cgi/doi/10.1542/ peds.2011-1330.

4. Salas P, Barrera P, González C, et al. Actualización en el diagnóstico y manejo de la Infección Urinaria en pediatría. Rev Chil Pediatr. 2012;83(3):269-78.

5. Shaikh N, Morone NE, Bost JE, Farrell MH. Prevalence of urinary tract infection in childhood: A meta-analysis. Pediatr Infect Dis J. 2008;27(4):302-8.

6. González Rodríguez JD, Rodríguez Fernández LM. Infección de Vías Urinarias en la infancia. Protoc diagn ter pediatr [Internet]. 2014;1(1):91-108. Available from: http://www.aeped.es/sites/ default/files/documentos/07_infeccion_ vias_urinarias.pdf.

7. Alarcon Alacio MT, Justa Roldan ML. Bacteriuria asintomática. Protoc diagn ter pediatr [Internet]. 2014;(1):109-17. Available from: http://www.aeped.es/ protocolos/

8. Etoubleau C, Reveret M, Brouet D, et al. Moving from Bag to Catheter for Urine Collection in Non-ToiletTrained Children Suspected of Having Urinary Tract Infection: A Paired Comparison of Urine Cultures. J Pediatr. 2009;154(6):803-6.

9. Hernangómez Vázquez S, Oñoro G, De La Torre Espí M, Martín Díaz MJ, Novoa-Carballal R, Molina Cabañero JC. Complicaciones del cateterismo vesical realizado en un servicio de urgencias para obtener una muestra de orina. An Pediatr. 2011;75(4):253-8

10. Doern CD, Richardson SE. Diagnosis of Urinary Tract Infections in Children. Kraft CS, editor. J Clin Microbiol [Internet]. 2016;54(9):2233-42. Available from: http://dx.doi.org/10.1016/j. jacr.2017.02.028.

11. Vaillancourt S, McGillivray D, Zhang $\mathrm{X}$, Kramer MS. To Clean or Not to Clean: Effect on Contamination Rates in Midstream Urine Collections in Toilet-Trained Children. Pediatrics. 2007;119(6):e1288-93.

12. Swerkersson S, Jodal U, Åhrén C, Sixt R, Stokland E, Hansson S. Urinary tract infection in infants: the significance of low bacterial count. Pediatr Nephrol. 2016;31(2):239-45.

13. Shaikh N, Borrell JL, Evron J, Leeflang MM. Procalcitonin, C-reactive protein, and erythrocyte sedimentation rate for the diagnosis of acute pyelonephritis in children. Cochrane Database Syst Rev [Internet]. 2015;(1). Available from: www. cochranelibrary.com.

14. Schnadower D, Kuppermann N, Macias CG, et al. Febrile Infants With Urinary Tract Infections at Very Low Risk for Adverse Events and Bacteremia.
Pediatrics. 2010;126(6):1074-83.

15. Silva OF, Cifuentes DM, Pinto CME. Resultados de la vigilancia de susceptibilidad antimicrobiana en Chile: Consolidando una red. Rev Chilena Infectol. 2013;28(1):19-27.

16. Fitzgerald A, Mori R, Lakhanpaul M, Tullus K. Antibiotics for treating lower urinary tract infection in children. Cochrane Database Syst Rev [Internet]. 2012;(8). Available from: http://doi.wiley. com/10.1002/14651858.CD006857.pub2.

17. Strohmeier Y, Hodson EM, Willis NS, Webster AC, Craig JC. Antibiotics for acute pyelonephritis in children. Cochrane Database Syst Rev [Internet]. 2014;(7). Available from: http://doi.wiley. com/10.1002/14651858.CD003772.pub4

18. McMullan BJ, Andresen D, Blyth CC, et al. Antibiotic duration and timing of the switch from intravenous to oral route for bacterial infections in children: systematic review and guidelines. Lancet Infect Dis [Internet]. 2016;16(8):e139-52. Available from: https://linkinghub.elsevier.com/ retrieve/pii/S147330991630024X.

19. Schroeder AR, Shen MW, Biondi EA, et al. Bacteraemic urinary tract infection: management and outcomes in young infants. Arch Dis Child [Internet]. 2016;101(2):125-30. Available from: http://adc.bmj.com/lookup/doi/10.1136/ archdischild-2014-307997.

20. Riordan A. 5, 7, 10 or 14 days: Appropriate duration of treatment for bacteraemia or an example of "antimicrobial bingo"? Arch Dis Child. 2016;101(2):117-8.

21. Robinson JL, Le Saux N. Management of urinary tract infections in children in an era of increasing antimicrobial resistance. Expert Rev Anti Infect Ther [Internet]. 2016;14(9):809-16. Available from: https://www.tandfonline.com/doi/full/10. 1080/14787210.2016.1206816. 